\title{
Sympositum (II)
}

\section{Clinical Aspects of Intracranial Tumors}

\section{Chairman :}

Prof. K. Tanaka

Juntendo Med. College

\section{H7. Surgical Aspects of Brain Tumors.}

\author{
C. ARAKI
}

The 1st Surg. Div., Kyoto Univ. Med. School

\begin{abstract}
Gliomas
1) 280 gliomas in the author's' clinic, which were histologically verified, consisted of 101 astrocytomas, 91 glioblastomas, 47 ependynomas and smaller groups of other gliomas.

2) Some cases are presented in which the tumor was a benign astrocytoma at the first operation, whereas it was a malignant astrocytoma or a glioblastoma at the second operation 2 or 3 years later.

3 ) Results of operations. Immediate mortality in gliomas of all kinds and locations was $36 \%$. It was rather lower in grossly total removal than in partial resection. Although discomforts of patients are relieved by internal decompression for some time, the remote results of the operations are not satisfactory, there being no remarkable difference in result between total, subtotal and partial removal. The same is true of well demarcated gliomas, which are apt to be removed totally. 4) Removal of a mural nodule in a cerebral cystic glioma usually does not mean a radical procedure.

5) Even in children under the age of 15 , cerebral glioblastomas are not unusual, the operative results being poor.

6) From the cases of glioma without accompanying increased intracranial pressure it may be assumed that gliomas found at the early stage can not always be removed radically.

7) After internal decompression for cerebral glioblastoma, angiography is to be done to see whether the decompression is actually effected or not.

8) Postoperative X-ray irradiation is recommended for glioblastoma, since


there are some, even if not many, cases in which such a procedure seemed effective. Meningioma

1) In 133 cases of meningioma there are at least 21 cases (16\%) of malignant type.

2) Survival for more than 5 years is very often even in cases of incomplete removal. In these cases it would be wise to make angiography after the operation to see exactly the extent of the remaining tumor.

3) In carotid angiography it is shown that feeding arteries of meningiomas are derived to greater extent from internal carotid than from external. Surgeons should be aware of such artery or arteries before attacking the tumor.

4) Preoperative attacks of convulsions often disappear or markedly decrease after operation.

Acoustic neurinoma

In our experiences extracapsular total extirpation of the tumor is accompanied neither by higher mortality nor by better neurological states soon or late after operation.

Hypophyseal adenoma

1) Even after grossly complete curettage of the tumor the upward shift of anterior cerebral artery in angiography returns usually not remarkably.

2) Grade 1 and 2 of hypothalamic extension were divided according to the changes in iodized oil ventriculography, carotid angiography and neurological states. Immediate operation mortality is highest (50\%) in grade 2 cases and lowest $(10 \%)$ in non-extension cases.

Pineal tumors

Mortality of extirpation of pineal tumors is lowest in teratomas and highest in gliomas. Therefore, preoperative differentiation of gliomas from teratomas or pinealomas is desired. In this connection some characteristic changes in iodized oil ventriculography, carotid angiography and neurological states are suggested.

\title{
H2. A Statistical Observation of Intracranial Tumors.
}

\author{
Keiji Sano \\ Dept. of Neurosurg., Univ. of Tokyo
}

This report consists of statistics on 1546 cases of intracranial tumors (neoplastic tumors: 1354, non-neoplastic tumors :192) which were experienced in our department until December, 1960 (Table 1).

Figure 1 shows age and sex distribution of 1354 cases of neoplastic tumors. There is a slight preponderance of males over females (approximately $1.3: 1$ ). Of these, cases below the age of 15 years are seen in $17 \%$, the sex ratio being also $1.3: 1$ (male : female). Age distribution of these cases divided into supra- 